\title{
First results from DAMA/LIBRA
}

\section{R. Bernabei, P. Belli*, F. Montecchia, F. Nozzoli}

Dip. di Fisica, Università di Roma “Tor Vergata" and INFN, sez. Roma “Tor Vergata”, I-00133

Rome, Italy

E-mail: pierluigi.bellieroma2.infn.it

\section{F. Cappella, A. d'Angelo, A. Incicchitti, D. Prosperi}

Dip. di Fisica, Università di Roma "La Sapienza” and INFN, sez. Roma "La Sapienza”, I-00185 Rome, Italy

\section{R. Cerulli}

Laboratori Nazionali del Gran Sasso, I.N.F.N., I-67010 Assergi (Aq), Italy

\section{C.J. Dai, H.L. He, H.H. Kuang, J.M. Ma, X.D. Sheng, Z.P. Ye}

IHEP, Chinese Academy, P.O. Box 918/3, Beijing 100039, China

\begin{abstract}
The highly radiopure $\simeq 250 \mathrm{~kg} \mathrm{NaI(Tl)} \mathrm{DAMA/LIBRA} \mathrm{set-up} \mathrm{is} \mathrm{running} \mathrm{at} \mathrm{the} \mathrm{Gran} \mathrm{Sasso} \mathrm{Na-}$ tional Laboratory of the I.N.F.N.. The first results obtained by exploiting over four annual cycles the model independent annual modulation signature for Dark Matter (DM) particles confirm those of the former DAMA/NaI. In particular, the DAMA/NaI and DAMA/LIBRA data satisfy all the many peculiarities of the DM annual modulation signature. Neither systematic effects nor side reactions able to account for the observed modulation amplitude and to contemporaneously satisfy all the several requirements of the DM signature are available. Considering the former DAMA/NaI and the present DAMA/LIBRA data all together (total exposure 0.82 ton $\times \mathrm{yr}$ ), the presence of Dark Matter particles in the galactic halo is supported at $8.2 \sigma$ C.L.
\end{abstract}

Identification of dark matter 2008

August 18-22, 2008

Stockholm, Sweden

* Speaker. 


\section{Introduction}

The former DAMA/NaI [1, 2, 3, 4, 5], and the present DAMA/LIBRA set-ups [6, 7], have been developed inside the DAMA project. The DAMA project, mainly based on the development and use of low background scintillators, is also composed by other low background set-ups [8]: i) $\mathrm{DAMA} / \mathrm{LXe}(\simeq 6.5$ pure liquid Xenon scintillator); ii) DAMA/R\&D, devoted to tests on prototypes and small scale experiments; iii) DAMA/Ge detector for sample measurements. The former DAMA/NaI experiment has achieved many competitive results on rare processes and has pointed out a model independent evidence for the presence of DM particles in the galactic halo with high C.L.. In 1996 - while running the DAMA/NaI set-up - DAMA proposed to INFN to develop and to build a one ton set-up to further investigate Dark Matter particles and other rare processes. A second generation $\mathrm{R} \& \mathrm{D}$ project was funded at that time to develop new highly radiopure $\mathrm{NaI}(\mathrm{Tl})$ detectors towards the achievement of an intermediate step: the $\simeq 250 \mathrm{~kg}$ highly radiopure $\mathrm{NaI}(\mathrm{Tl})$ DAMA/LIBRA (Large sodium Iodide Bulk for RAre processes) set-up, which is now in data taking.

The DAMA/NaI and the DAMA/LIBRA experiments have the main aim to perform a direct detection of Dark Matter (DM) particles in the galactic halo by exploiting the model independent annual modulation signature (originally suggested in ref. [9]). In particular, the DM annual modulation signature exploits the correlation between the Earth velocity in the galactic halo with the number of events induced by DM particles in a suitable low background set-up placed deep underground. In fact, as a consequence of its annual revolution, the Earth should be crossed by a larger flux of DM particles around roughly June $2^{\text {nd }}$ (when its rotational velocity is summed to the one of the solar system with respect to the Galaxy) and by a smaller one around roughly December $2^{\text {nd }}$ (when the two velocities are subtracted). Thus, the contribution of the signal to the counting rate in the $k$-th energy interval can be written as (see e.g. ref.[2]): $S_{k}=S_{0, k}+S_{m, k} \cos \omega\left(t-t_{0}\right)$, where: i) $S_{0, k}$ is the constant part of the signal; ii) $S_{m, k}$ is the modulation amplitude; iii) $\omega=\frac{2 \pi}{T}$ with period $T$; iv) $t_{0}$ is the phase.

This annual modulation signature is very distinctive since the effect induced by DM particles must simultaneously satisfy all the following requirements: 1) the rate must contain a component modulated according to a cosine function; 2) with one year period; 3) with a phase roughly around June $2^{\text {nd }}$ in case of usually adopted halo models (slight variations may occur in case of presence of non thermalized DM components in the halo); 4) this modulation must be present only in a well-defined low energy range, where DM particles can induce signals; 5) it must be present only in those events where just a single detector, among all the available ones in the used set-up, actually "fires" (single-hit events), since the probability that DM particles experience multiple interactions is negligible; 6) the modulation amplitude in the region of maximal sensitivity has to be $\lesssim 7 \%$ in case of usually adopted halo distributions, but it may be significantly larger in case of some particular scenarios such as e.g. those of ref.[10]. To mimic such a signature spurious effects or side reactions should be able not only to account for the observed modulation amplitude but also to contemporaneously satisfy all the requirements of the signature; none of these has been found or suggested by anyone over more than a decade (see e.g. ref. [2,7] and references therein).

The corollary question about the nature of the DM particle(s) detected by the annual modulation signature and the related astrophysical, nuclear and particle Physics scenarios requires subse- 
quent model dependent corollary analyses as those performed e.g. in refs. [2, 3, 4]; an update is in preparation. One should stress that it does not exist any approach to investigate the nature of the candidate in the direct and indirect DM searches which can offer these information independently on assumed astrophysical, nuclear and particle Physics scenarios.

The DAMA/LIBRA set-up, its main features and radiopurity have been discussed in details in ref. [6], while the model independent experimental results obtained by DAMA/LIBRA (exposure of 0.53 ton $\times$ yr over 4 annual cycles) and those combined with DAMA/NaI (exposure of 0.29 ton $\times$ yr over 7 annual cycles) have been discussed in details in ref. [7] and are shortly reminded in some aspects in the following.

\section{The model-independent experimental results}

DAMA/LIBRA started the first operations in March 2003. The data taking for the investigation of the annual modulation signature has been started on September 9, 2003; this has allowed the decay of medium half-life isotopes. Moreover, it is worth noting that one of the more external detectors has been put out of operation few months after installation because of a PMT break. Since the disinstallation and re-installation of this detector would have required the opening of the set-up, we have delayed this; the related procedure has been performed in September 2008, when realizing an upgrade of the set-up. Therefore, the exposed mass in the first four annual cycles is $232.8 \mathrm{~kg}$.

A model-independent investigation of the annual modulation signature has been carried out by exploiting the time behaviour of the residual rates of the single-hit events in the lowest energy regions. Fig. 1 shows the time behaviour of the model-independent residual rates for single-hit events collected by the former DAMA/NaI experiment and by the present DAMA/LIBRA in the (2-4), (2-5) and (2-6) keV energy intervals. The cumulative analysis of the single-hit residual rate favours the presence of a modulated cosine-like behaviour with proper features at $8.2 \sigma$ C.L. [7].

The same data of Fig.1 have also been investigated by a Fourier analysis (see ref. [7] for details). A clear peak corresponding to a period of 1 year is evident in the lowest (2-6) keV energy interval, while in the $(6-14) \mathrm{keV}$ energy interval only aliasing peaks are instead present. Thus, a clear modulation is present in the lowest energy interval, while it is absent just above. In fact, the best fitted modulation amplitude in the $(6-14) \mathrm{keV}$ energy region is well compatible with zero: $(0.0004 \pm 0.0010) \mathrm{cpd} / \mathrm{kg} / \mathrm{keV}[7]$.

To verify absence of annual modulation in other energy regions and, thus, to also verify the absence of any significant background modulation, the whole energy distribution has been investigated. In particular, we have investigated the rate integrated above $90 \mathrm{keV}, \mathrm{R}_{90}$, as a function of the time. The result excludes any significant background variation. Moreover, fitting the time behaviour of $\mathrm{R}_{90}$ with phase and period as for DM particles, a modulation amplitude compatible with zero is found in each running period: $-(0.05 \pm 0.19) \mathrm{cpd} / \mathrm{kg},-(0.12 \pm 0.19) \mathrm{cpd} / \mathrm{kg}$, $-(0.13 \pm 0.18) \mathrm{cpd} / \mathrm{kg}$, and $(0.15 \pm 0.17) \mathrm{cpd} / \mathrm{kg}$ for DAMA/LIBRA-1 to -4 annual cycles, respectively. This excludes the presence of a background modulation in the whole energy spectrum at a level much lower than the effect found in the lowest energy region for the single-hit events; in fact, otherwise - considering the $\mathrm{R}_{90}$ mean values - a modulation amplitude of order of tens $\mathrm{cpd} / \mathrm{kg}$, that $\simeq 100 \sigma$ far away from the measured value, would be present. 


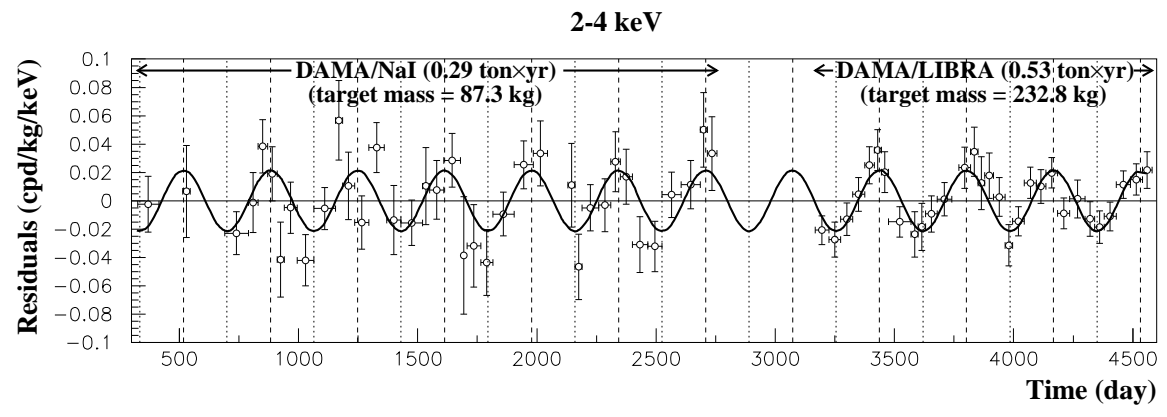

2-5 keV

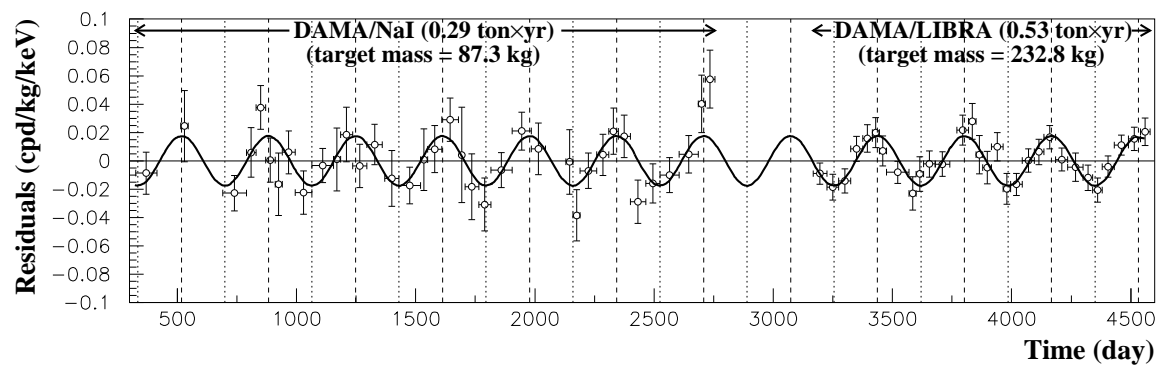

2-6 keV

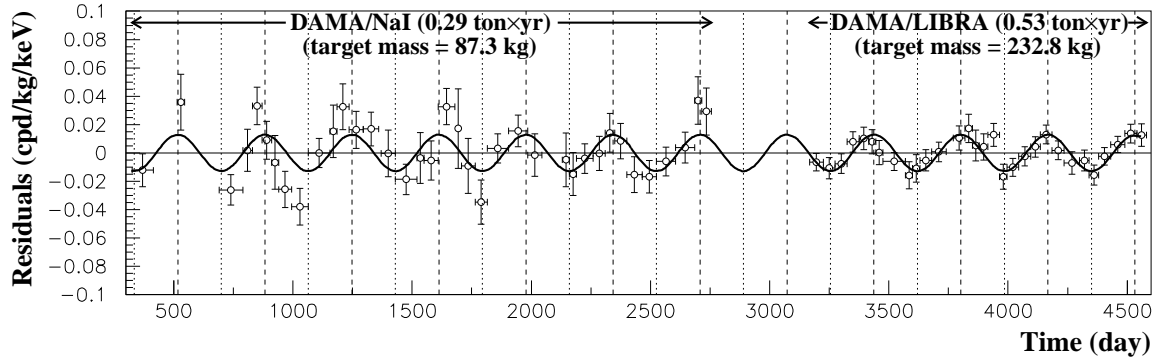

Figure 1: Model-independent residual rate of the single-hit scintillation events, measured by DAMA/NaI and DAMA/LIBRA in the $(2-4),(2-5)$ and $(2-6) \mathrm{keV}$ energy intervals as a function of the time. The zero of the time scale is January $1^{\text {st }}$ of the first year of data taking of the former DAMA/NaI experiment. The experimental points present the errors as vertical bars and the associated time bin width as horizontal bars. The superimposed curves represent the cosinusoidal functions behaviours $A \cos \omega\left(t-t_{0}\right)$ with a period $T=\frac{2 \pi}{\omega}=1 \mathrm{yr}$, with a phase $t_{0}=152.5$ day (June $2^{\text {nd }}$ ) and with modulation amplitudes, $A$, equal to the central values obtained by best fit over the whole data, that is: $(0.0215 \pm 0.0026) \mathrm{cpd} / \mathrm{kg} / \mathrm{keV},(0.0176 \pm 0.0020)$ $\mathrm{cpd} / \mathrm{kg} / \mathrm{keV}$ and $(0.0129 \pm 0.0016) \mathrm{cpd} / \mathrm{kg} / \mathrm{keV}$ for the $(2-4) \mathrm{keV}$, for the $(2-5) \mathrm{keV}$ and for the $(2-6)$ $\mathrm{keV}$ energy intervals, respectively. When the period and the phase parameters are released in the fit, values well compatible with those expected for DM particle induced effect are obtained [7]. The dashed vertical lines correspond to the maximum of the signal (June $2^{\text {nd }}$ ), while the dotted vertical lines correspond to the minimum. The total exposure is 0.82 ton $\times$ yr. For details see [7].

A further relevant investigation has been done by applying the same hardware and software procedures, used to acquire and to analyse the single-hit residual rate, to the multiple-hits one. In fact, since the probability that a DM particle interacts in more than one detector is negligible, a DM signal can be present just in the single-hit residual rate. Thus, this allows the test of the background behaviour in the same energy interval of the observed positive effect. We remind that similar investigations have already been performed for the last two annual cycles of the DAMA/NaI 


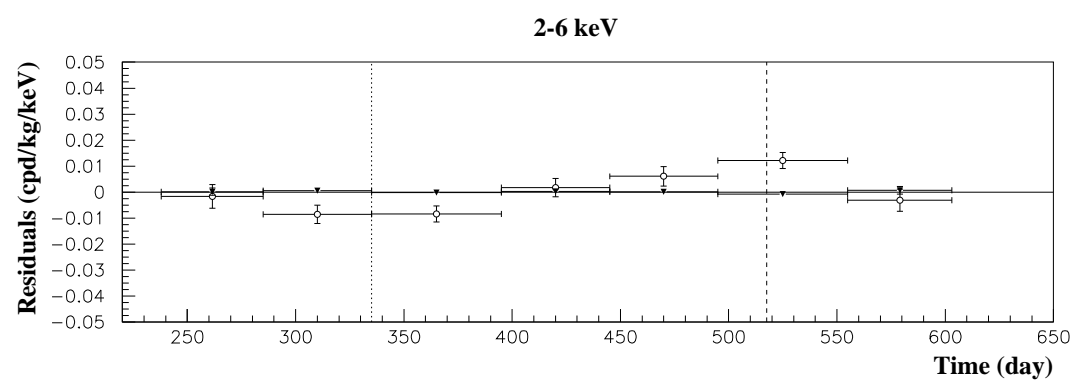

Figure 2: Experimental residual rates over the four DAMA/LIBRA annual cycles for single-hit events (open circles) - class of events to which DM events belong - and for multiple-hits events (filled triangles) - class of events to which DM events do not belong - in the (2-6) keV energy intervals. They have been obtained by considering for each class of events the data as collected in a single annual cycle and by using in both cases the same identical hardware and the same identical software procedures. The initial time of the scale is taken on August $7^{\text {th }}$. The experimental points present the errors as vertical bars and the associated time bin width as horizontal bars. See ref. [7].

experiment [2]. Thus, in Fig. 2 the residual rates of the single-hit events measured over the four DAMA/LIBRA annual cycles are reported, as collected in a single annual cycle, together with the residual rates of the multiple-hits events, in the considered energy interval. As already observed, a clear modulation is present in the single-hit events, while the fitted modulation amplitudes for the multiple-hits residual rate are well compatible with zero: $-(0.0004 \pm 0.0008) \mathrm{cpd} / \mathrm{kg} / \mathrm{keV}$, $-(0.0005 \pm 0.0007) \mathrm{cpd} / \mathrm{kg} / \mathrm{keV}$, and $-(0.0004 \pm 0.0006) \mathrm{cpd} / \mathrm{kg} / \mathrm{keV}$ in the energy regions $(2-$ $4),(2-5)$ and $(2-6) \mathrm{keV}$, respectively. Thus, again evidence of annual modulation with proper features as required by the DM annual modulation signature is present in the single-hit residuals (events class to which the DM particle induced events belong), while it is absent in the multiplehits residual rate (event class to which only background events belong). Since the same identical hardware and the same identical software procedures have been used to analyse the two classes of events, the obtained result offers an additional strong support for the presence of a DM particle component in the galactic halo further excluding any side effect either from hardware or from software procedures or from background.

The annual modulation present at low energy can also be shown by depicting the differential modulation amplitudes, $S_{m, k}$ values, as a function of the energy (see ref. [7]), obtained by maximum likelihood method considering $T=1 \mathrm{yr}$ and $t_{0}=152.5$ day. Positive signal is present in the (26) $\mathrm{keV}$ energy interval, while $S_{m, k}$ values are compatible with zero just above [7]. It has also been verified that the measured modulation amplitudes are statistically well distributed in all the crystals, in all the annual cycles and in the energy bins; these and other discussions can be found in ref. [7]. Moreover, the assumption of a phase $t_{0}=152.5$ day in the maximum likelihood procedure has been released, alternatively writing the signal as: $S_{k}=S_{0, k}+S_{m, k} \cos \omega\left(t-t_{0}\right)+Z_{m, k} \sin \omega(t-$ $\left.t_{0}\right)=S_{0, k}+Y_{m, k} \cos \omega\left(t-t^{*}\right)$. Fig. 3-left shows the $2 \sigma$ contours in the plane $\left(S_{m}, Z_{m}\right)$ for the (2-6) $\mathrm{keV}$ and (6-14) keV energy intervals and Fig. 3-right shows, instead, those in the plane $\left(Y_{m}, t^{*}\right)$. These results confirm those achieved above by analysing the residuals. In particular, a modulation amplitude is present in the lower energy intervals and the period and the phase agree with those expected for DM induced signals. Several other analyses have been performed; for more 

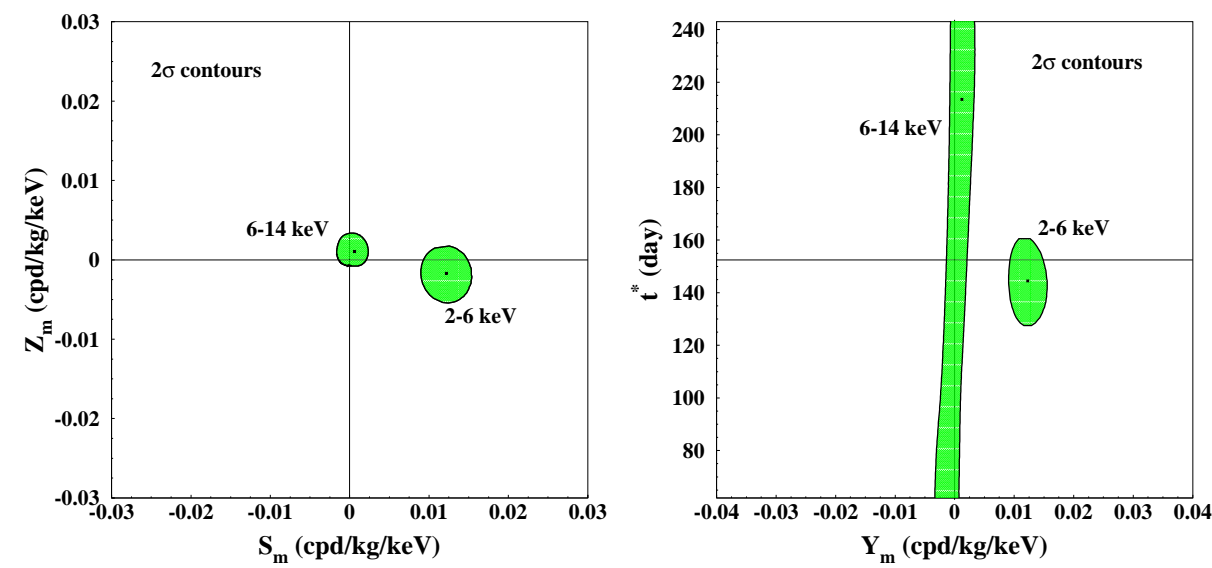

Figure 3: $2 \sigma$ contours in the plane $\left(S_{m}, Z_{m}\right)$ (left) and in the plane $\left(Y_{m}, t^{*}\right)(r i g h t)$ for the (2-6) keV and (614) $\mathrm{keV}$ energy intervals. The contours have been obtained by the maximum likelihood method, considering the seven annual cycles of DAMA/NaI and the four annual cycles of DAMA/LIBRA all together. The best fit values for the (2-6) keV energy interval are $\left(1 \sigma\right.$ errors): $S_{m}=(0.0122 \pm 0.0016) \mathrm{cpd} / \mathrm{kg} / \mathrm{keV} ; Z_{m}=$ $-(0.0019 \pm 0.0017) \mathrm{cpd} / \mathrm{kg} / \mathrm{keV} ; Y_{m}=(0.0123 \pm 0.0016) \mathrm{cpd} / \mathrm{kg} / \mathrm{keV} ; t^{*}=(144.0 \pm 7.5)$ day; while for the (6-14) $\mathrm{keV}$ energy interval are: $S_{m}=(0.0005 \pm 0.0010) \mathrm{cpd} / \mathrm{kg} / \mathrm{keV} ; Z_{m}=(0.0011 \pm 0.0012) \mathrm{cpd} / \mathrm{kg} / \mathrm{keV}$; $Y_{m}=(0.0012 \pm 0.0011) \mathrm{cpd} / \mathrm{kg} / \mathrm{keV}$ and $t^{*}$ obviously not determined. See ref. [7] for details.

information see ref. [6, 7].

In conclusion, also the data of the first four annual cycles of DAMA/LIBRA as previously those of DAMA/NaI fulfil all the requirements of the DM annual modulation signature. As previously done for DAMA/NaI [2], careful investigations on absence of any significant systematics or side reaction effect have been quantitatively carried out and reported in details in ref. [7]. No modulation has been found in any possible source of systematics or side reactions for DAMA/LIBRA as well; thus, cautious upper limits (90\% C.L.) on the possible contributions to the DAMA/LIBRA measured modulation amplitude have been estimated [7]. In addition, it is worth stressing that neither systematics nor side reaction effect - able to contemporaneously satisfy all the requirements of the signature and to account for the measured modulation amplitude - have been found or suggested by anyone over more than a decade ${ }^{1}$. For detailed quantitative discussions on all the related topics and for results see ref. [7] and refs. therein.

\section{Conclusions}

The model independent results achieved by the second generation DAMA/LIBRA set-up confirms the former DAMA/NaI result; in particular, considering the DAMA/NaI and the DAMA/LIBRA data (total exposure 0.82 ton $\times y r$ ), the presence of Dark Matter particles in the galactic halo is supported at $8.2 \sigma$ C.L.. Deep quantitative analyses exclude any effect either from systematics or from

\footnotetext{
${ }^{1}$ We take this occasion to also point out to the attention of the reader that e.g. the DM annual modulation indeed is not a "seasonal" variation and it is not a "winter-summer" effect, as sometimes naively mentioned. In fact, the DM annual modulation is not related to the relative Sun position, but it is related to the Earth velocity in the galactic frame. Thus, in particular, the phase of the DM annual modulation (roughly $2^{\text {nd }}$ June) is well different than those of physical quantities (such as temperature of atmosphere, pressure, other meteorological parameters, cosmic rays flux, ...) instead correlated with seasons.
} 
side processes able to account for the observed modulation amplitude and to contemporaneously satisfy all the requirements of the signature $[2,7]$.

Corollary investigations on the nature of the DM candidate particle and on related astrophysical, nuclear and particle Physics scenarios are in progress. As widely discussed elsewhere, these investigations are instead model-dependent and - considering the large uncertainties which exist on the astrophysical, nuclear and particle physics assumptions and on the theoretical and experimental parameters needed in the calculations - have no general meaning (as it is also the case of exclusion plots and of the DM particle parameters evaluated in indirect detection experiments).

It is worth noting that no experiment exists whose result can be directly compared in a model independent way with the DAMA/NaI and DAMA/LIBRA results. We have addressed some of the related arguments e.g. in refs. [2, 3, 4, 6, 7, 11]; other ones are available in literature.

Finally, a larger exposure with DAMA/LIBRA (and with the possible DAMA/1ton, which is at $\mathrm{R} \& \mathrm{D}$ stage) and the possible lowering of the $2 \mathrm{keV}$ energy threshold will improve the corollary information on the nature of the DM candidate particle(s) and on the various related astrophysical, nuclear and particle Physics scenarios. Moreover, it will also allow the investigation - with very high sensitivity - of other DM features, of second order effects and of several rare processes other than DM.

\section{References}

[1] R. Bernabei et al., Phys. Lett. B 389 (1996) 757; Phys. Lett. B 424 (1998) 195; Phys. Lett. B 450 (1999) 448; Phys. Lett. B 480 (2000) 23; Phys. Lett. B 509 (2001) 197; Eur. Phys. J. C 23 (2002) 61; P. Belli et al., Phys. Rev. D 61 (2000) 023512; Phys. Rev. D 66 (2002) 043503.

[2] R. Bernabei et al., La Rivista del Nuovo Cimento 26 n.1 (2003) 1-73; R. Bernabei et al., Int. J. Mod. Phys. D 13 (2004) 2127.

[3] R. Bernabei et al., Eur. Phys. J. C 47 (2006) 263.

[4] R. Bernabei et al., Il Nuovo Cim. A 112 (1999) 545; Eur. Phys. J. C 18 (2000) 283; Int. J. Mod. Phys. A 21 (2006) 1445; Int. J. Mod. Phys. A 22 (2007) 3155; Eur. Phys. J. C 53 (2008) 205; Phys. Rev. D 77 (2008) 023506; Mod. Phys. Lett. A 23 (2008) 2125.

[5] R. Bernabei et al., Phys. Lett. B 408 (1997) 439; Phys. Rev. Lett. 83 (1999) 4918; Il Nuovo Cimento A 112 (1999) 1541; P. Belli et al., Phys. Lett. B 460 (1999) 236; Phys. Rev. C 60 (1999) 065501; R. Bernabei et al., Phys. Lett. B 515 (2001) 6; F. Cappella et al., Eur. Phys. J.-direct C 14 (2002) 1; R. Bernabei et al., Eur. Phys. J. A 23 (2005) 7; Eur. Phys. J. A 24 (2005) 51.

[6] R. Bernabei et al., Nucl. Instr. \& Meth. A 592 (2008) 297.

[7] R. Bernabei et al., Eur. Phys. J. C 56 (2008) 333.

[8] For some references see the DAMA web page: http://people.roma2.infn.it/dama/

[9] K.A. Drukier et al., Phys. Rev. D 33 (1986) 3495; K. Freese et al., Phys. Rev. D 37 (1988) 3388.

[10] D. Smith and N. Weiner, Phys. Rev. D 64 (2001) 043502; D. Tucker-Smith and N. Weiner, Phys. Rev. D 72 (2005) 063509.

[11] R. Bernabei et al., arXiv:0806.0011[astro-ph]. 